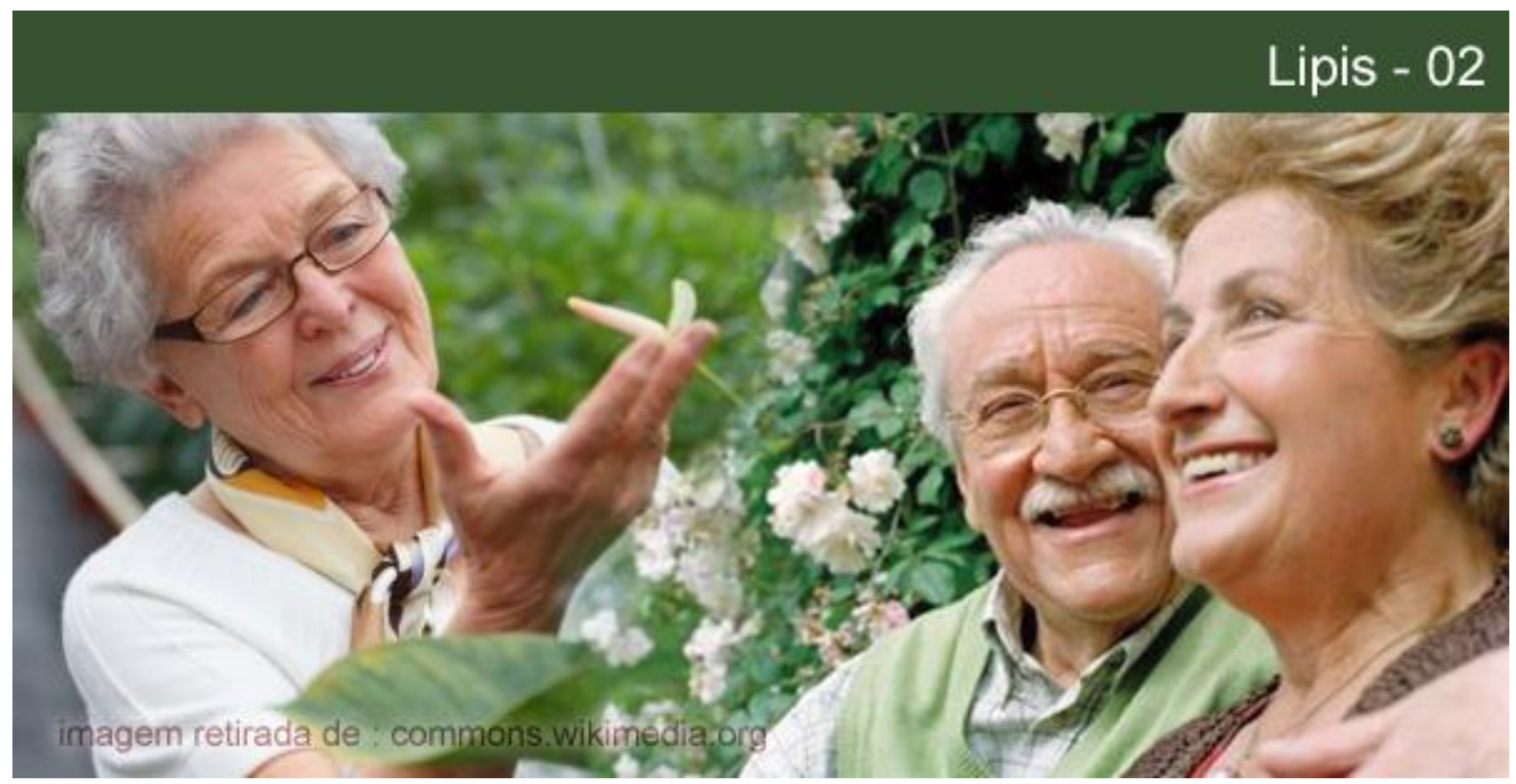

\title{
REDES DE AMIGOS E VIZINHANÇA COMO FATOR DE PROTEÇÃO SOCIAL PARA PESSOAS IDOSAS ISOLADAS: ESTUDO PILOTO EM ALDEIAS CONCELHO DA GUARDA
}

\section{Ricardo Pocinho}

Doutor em Psicogerontologia pela Universidade de Valencia- Espanha. Secretário, Investigador e Professor na Escola Superior de Tecnologia da Saúde de Coimbra. E-mail: pocinho@hotmail.com.

\section{Jorge Castro}

Licenciado em Gerontologia Social - Gerontólogo Social na Residência Sénior, Fundação Augusto Gil, Guarda. Membro da Associação Nacional de Gerontologia Social (ANGES). E-mail: jorgemiguelalmeidacastro@gmail.com.

\section{Gisela Santos}

Licenciada em Psicologia e Mestre em Psicologia Clínica e Saúde. Psicogerontologia Clínica pela Faculdade de Psicologia e de Ciências da Educação da Universidade de Coimbra. E-mail: xiza_santos@hotmail.com.

\section{Carlos Mendes Rosa}

Psicólogo. Doutorando do Programa de Pós-Graduação em Psicologia da PUC-Rio. Pesquisador Associado do Laboratório Interdisciplinar de Pesquisa e Intervenção Social da PUC-Rio. E-mail: carlosmendesrosa@gmail.com.

Resumo: O presente estudo pretende compreender a importância da rede de amigos e vizinhança na percepção de segurança e de proteção por parte das pessoas idosas residentes em contexto rural. A amostra foi constituída por 50 pessoas idosas residentes em meio rural, em diversas aldeias do Concelho da Guarda. De forma a analisar o objeto proposto, recorreu-se à Escala Satisfação com o Suporte Social. Os resultados obtidos no presente estudo indicam que os vizinhos e/ou amigos são um bom nível de apoio para as pessoas idosas isoladas.

Palavras-chave: Meio rural. Redes de suporte. Vizinhança. Isolamento.

\section{FRIENDS AND NEIGHBOURHOOD NETWORKS AS SOCIAL PROTECTION FACTOR FOR ELDERLY ISOLATED: PILOT STUDY IN CONCELHO DA GUARDA VILLAGES}

\section{POLÊM!CA | Revista Eletronica da Uej}


Abstratct: This study aims to understand the importance of the network of friends and neighbors in the security perception and of protection of older persons living in rural context. The sample consisted of 50 elderly people living in rural areas, in several villages of the municipality of Guarda. In order to analyze the proposed object resorted to Scale Satisfaction with Social Support. The results obtained in this study indicate that the neighbors and / or friends are a good level of support for isolated older people.

Keywords: Rural Environment. Support networks. Neighborhood. Isolation.

\section{Introdução}

A visão relativa ao processo de envelhecimento tem vindo a sofrer profundas alterações ao longo do tempo em diversas áreas da vida humana. Tornou-se, no século XXI, o fenômeno mais relevante e o que mais questões e desafios coloca na sociedade. $\mathrm{O}$ envelhecimento projeta relevo e uma prioridade indiscutível, devido ao aumento do número de idosos, e sobretudo, dos muito idosos. Isto mesmo vem no sentido daquilo que Varues (1983 apud FIGUEIREDO, 2014, p.34) afirma: “o processo de envelhecimento tem sobretudo uma dimensão existencial, modificando a relação do homem com o tempo". Encetar conhecimento/estudo sobre este fenômeno, é de grande importância, uma vez que no último CENSOS 2011 (INE, 2011) estão bem patentes os números da população portuguesa; 19\% apresenta uma idade superior a 65 anos (cerca de 2 milhões de idosos), contrariamente aos $15 \%$ do grupo etário dos mais jovens (0-14 anos) e uma esperança média de vida à nascença de 79,2 anos. Havia em 2011, um índice de envelhecimento de 128, ou seja, por cada 100 jovens, existem 128 idosos. Estamos perante um aumento exponencial da população idosa, neste sentido o envelhecimento é um dos desafios mais importantes deste século, com tendência para o seu aumento progressivo. De acordo com as projeções realizadas pelo INE, o peso total da população idosa, no total da população, aumentará para os 32,3\% em 2060 (3 043 milhares) (INE, 2011). Esta mesma dinâmica populacional aponta para uma transição demográfica sem precedentes na história, uma vez que, o envelhecimento populacional que se tem vindo a verificar nos últimos anos, acarreta, de forma explícita e concreta, a emergência de uma problematização social, através de uma subjugação dos indivíduos idosos a uma normatização que valoriza a independência e autonomia dos mesmos.

Atualmente verifica-se uma situação complexa onde os atuais problemas da sociedade não residem no envelhecimento nem no aumento da esperança de vida, mas sim no fato de a sociedade não se ter adaptado ao "inverno demográfico". São colocados novos desafios à população, no sentido do envelhecimento ser compreendido não como declínio físico, mas sim como uma conquista civilizacional, onde são necessárias sinergias para que este seja bem- 
sucedido e vivenciado com qualidade. Conscientes desta necessidade e da importância de investigação no contexto rural, o presente estudo pretende dar um contributo no conhecimento da importância da relação entre os amigos e vizinhança na rede social das pessoas idosas que vivem isoladas, e num contexto rural, percebendo qual o nível de satisfação que o suporte social pode proporcionar na terceira idade.

O contexto em que uma pessoa se relaciona interfere tanto nas relações sociais como no próprio processo de envelhecimento. De acordo com Lawton (1983 apud SEQUEIRA et al., 2002), o meio ambiente em que a pessoa idosa vive é, de fato, um agente relevante na promoção do seu bem-estar, sendo portanto esta relação idoso-ambiente uma relação sensível. Assim, é necessário o conhecimento de como ocorre o processo de envelhecimento em contexto rural, percebendo as suas especificidades, uma vez que o homem não é mais do que um elemento num conjunto em que o ambiente desempenha um papel importante no seu bemestar e na sua própria saúde. Cada vez mais caminhamos para um desinvestimento desta franja da população, sendo as características rurais e a própria pessoa idosa "carregada" com estereótipos negativos que nada traduz o que deveria ser um envelhecimento ativo.

Kroutse e Coward (1998, apud FONSECA, PAÚL, MARTIN \& AMADO, 2005) referem os mitos existentes em relação à vivência da "velhice no campo", sendo que se destaca o fato de as pessoas idosas terem um forte apoio de redes familiares e que os mesmos estão sempre disponíveis para lhes prestar os cuidados necessários. Segundo Monteiro (2012), a maioria das pessoas idosas que vivem em meio rural vivem, de fato, longe das famílias estando estas ausentes da vida do idoso. Perante este cenário, percebe-se facilmente que o envelhecimento em meio rural necessita de uma reflexão urgente, uma vez que a tendência é para que este cenário se acentue.

É necessário potenciar e criar condições para que as pessoas idosas permaneçam integradas socialmente e que ao mesmo tempo se possa garantir a sua presença na vida coletiva. Segundo Cabral e colaboradores (2013, p.19) “defender a inclusão social, significa defender a possibilidade de se continuar a participar nos processos de decisão coletiva e para esse fim é importante preservar uma vida ativa durante mais tempo". Os meios rurais podem funcionar como um bom promotor de autonomia na velhice, uma vez que um dos fatores que pode ser considerado de coesão social é o fato de as aldeias constituírem um conjunto de edifícios dissociados especificamente uns dos outros. Esta mesma característica possibilita um 
maior contato entre a população e uma maior interajuda perante os diversos elementos. Atualmente, o grande problema com o envelhecimento é perceber-se efetivamente qual o lugar da velhice na sociedade (CABRAL et al., 2013).

Quando estamos perante situações em que as pessoas idosas vivem sozinhas, as mesmas têm de recorrer a fontes de suporte social para suprimir diversas expectativas e necessidades. As redes de amigos e vizinhos são as mais comuns em contexto rural, onde existe um ambiente de coesão social e uma forte rede e aproximação entre os diversos elementos. Muitas vezes, é mesmo a única fonte de apoio para as pessoas idosas. Isto refletese nas palavras de Domingues (2012, p.179): a "diminuição da família, os amigos, vizinhos e as restantes pessoas da comunidade, constituem uma rede de suporte social", podendo ainda proporcionar um sentimento de reconhecimento social. Estas mesmas relações não só contribuem para a participação comunitária como ajudam a sustentar o múltiplo processo de envelhecimento.

A rede de amigos e vizinhança tem um número significativo de pessoas, bem como uma aproximação das pessoas idosas, principalmente em função dos novos grupos (e.g. associações, clubes, entre outros). Na amizade está intrinsecamente associado o caráter da afetividade, sendo que as relações de vizinhança podem unicamente limitar-se à assistência para o serviço. Fraquinella e Marcon (2011) realçam o fato de as pessoas de idade avançada verem nas amizades uma importância peculiar, pois nesta idade dispõem de muito tempo ocioso, facilitando o contato com os amigos. Ao contrário das redes familiares, mais de carácter involuntário, as de amigos e vizinhos, assumem-se como uma escolha voluntária, desempenhando um papel pequeno, mas fundamental na ajuda à pessoa idosa. Papel este muito associado a questões de relacionamento afetivo. Amigos e vizinhos até podem parecer a mesma coisa, mas existem diferenças entre eles no que respeita às funções e ao próprio processo de relacionamento. Neste sentido, existem tarefas específicas destinadas unicamente aos amigos, nomeadamente as atividades instrumentais: fazer compras; auxílio nas atividades domésticas, entre outras (ANDRADE, 2002 apud DOMINGUES, 2012). Este tipo de relacionamento entre amigos só é possível uma vez que os amigos partilham as mesmas dificuldades e limitações, gostos e interesses.

Diversos estudos referem que a amizade contribui para o bem-estar na velhice de várias formas, através do apoio e companheirismo na vida cotidiana, ajuda na manutenção da

\section{POLÊM!CA | Revista Eletronica da Ueij}


identidade em mudança e na adaptação à velhice. Quanto à relação com os vizinhos, os mesmos "facilitam a obtenção de ajuda informal e reduzem o isolamento social contribuindo assim para o envelhecimento bem-sucedido" (CABRAL et al., 2013, p.141). Para Fraquinella e colaboradores (2011) os longos períodos de convivência com os vizinhos são marcados por atividades de ajuda mútua de modo que estes indivíduos se tornam integrantes recíprocos da história de vida das pessoas idosas.

Com o processo de envelhecimento, o diâmetro de espaço de vida das pessoas idosas tende a diminuir. Para estas, a sua vizinhança é o seu principal ou único suporte/retaguarda de vida e segurança, podendo esta refletir-se em diversos efeitos, influenciando ao nível das condições socioeconómicas, integração social da vizinhança, serviços e recursos (OLASS \& BALFOUR, 2008 apud FERREIRA, 2010). Os relacionamentos entre vizinhos são marcados pela proximidade geográfica e ao mesmo tempo por uma troca habitual de relações que se estendeu ao longo de anos de convivência. É necessário valorizarem-se as redes de amigos e vizinhos, uma vez que o isolamento social provocado por falta de contatos e de reconhecimento social está associado à solidão, à depressão e até às doenças do foro mental (DOMINGUES et al., 2012).

Noutros tempos, as relações de vizinhança e de amizade eram mais vincadas e com um maior número de indivíduos. Atualmente, devido à desertificação das aldeias, este tipo de relações denota uma tendência a perder-se num futuro próximo.

\section{Metodologia}

\section{$\underline{\text { Amostra }}$}

A amostragem do presente estudo é de conveniência, uma vez que os casos escolhidos são os que o investigador teve à sua disposição. A amostra foi constituída por 50 pessoas idosas que vivem isoladas e em meio rural, residentes em diversas aldeias do Concelho da Guarda (Pêra do Moço, Aldeia do Bispo, Arrifana e Casal de Cinza).

Os participantes que constituíram a amostra deste estudo cumpriram os seguintes critérios de inclusão: idade igual ou superior a 65 anos; residência em meio rural e numa das aldeias do Concelho da Guarda; isoladas socialmente na maior parte do seu tempo.

\section{POLÊM!CA | Revista Eletronica da Ueij}




\section{Objetivos}

O presente estudo pretende efetuar uma análise da importância da rede de amigos e vizinhança. De forma mais detalhada, pretende-se perceber se os amigos e os vizinhos são um bom suporte para as pessoas idosas em contexto rural e se existe um sentimento de proteção e segurança relacionado com esta rede social de suporte.

Foram traçadas as seguintes hipóteses, provenientes da observação de fenômenos da realidade, da teoria ou de trabalhos realizados: as pessoas idosas apresentam uma boa satisfação com o suporte social (H1); as pessoas idosas não apresentam uma boa satisfação com o suporte social (H0); os amigos e os vizinhos são um bom suporte social para as pessoas idosas e os mesmos prestam o auxílio e a proteção necessária (H1); os amigos e os vizinhos não são um bom suporte social para as pessoas idosas (H0); as pessoas idosas residentes em meio rural (isolados), apresentam uma maior satisfação em relação aos amigos do que em relação à família (H1); as pessoas idosas residentes em meio rural (isoladas) apresentam uma menor satisfação em relação aos amigos do que em relação à família (H0).

\section{$\underline{\text { Instrumentos }}$}

Elaborou-se um protocolo de investigação constituído por um questionário sociodemográfico e pela Escala de Satisfação com o Suporte Social - ESSS (RIBEIRO, 1999).

O questionário sociodemográfico foi elaborado especificamente para esta investigação e contém questões relacionadas com o gênero, idade, estado civil, nível de escolaridade e, ainda, questões específicas direcionadas especialmente para o presente estudo: auxílio por parte dos vizinhos e/ou amigos, proteção e necessidades em termos de urgência.

A Escala de Satisfação com o Suporte Social, desenvolvida por Ribeiro (1999), tratase de uma escala multidimensional, de autopreenchimento, constituída por 15 itens, de autopreenchimento, de resposta tipo Likert. Tem como objetivo principal verificar/avaliar quatro fatores: "Satisfação com os Amigos" (SA), que mede a satisfação com as amizades/amigos dos indivíduos; "Intimidade" (IN), que mede a percepção da existência de suporte social íntimo; Satisfação com a Família" (SF), que mede a satisfação com o suporte social familiar; "Atividades Sociais" (AS), que mede a satisfação com as atividades sociais que os indivíduos realizam. A pontuação para a escala total consiste no somatório de todos os

\section{POLÊM!CA | Revista Eletronica da ver}


itens e pode variar entre 15 e 75 e à pontuação mais alta corresponde uma percepção de maior suporte social. Não há pontos de corte que possam ser considerados como deficitários. (RIBEIRO, 1999).

\section{Procedimentos}

Aquando da recolha dos dados, todos os participantes foram informados acerca dos objetivos do estudo, a forma de administração do questionário e a confidencialidade dos dados. O tempo médio de administração foi de 10 minutos.

O tratamento dos dados efetuou-se através do programa estatístico SPSS 20.0 (Statistical Package for Social Sciences). Foram realizadas análises estatísticas descritivas relativamente à componente sociodemográfica da amostra na determinação das frequências absolutas e relativas; medidas de tendência central - média, mediana, moda, desvio padrão, como medida de dispersão. De seguida, analisou-se a ESSS relativa às pontuações totais e pontuações das variáveis latentes.

\section{Resultados}

\section{$\underline{\text { Análise descritiva }}$}

A amostra é constituída por 50 indivíduos de ambos os gêneros, com idades compreendidas entre os 67 anos e os 100 anos, $(\mathrm{M}=82.5)$ (c.f. Tabela 1). A amostra é maioritariamente feminina, como se pode observar através da Tabela 2.

Tabela - 1: Caracterização da amostra em relação à idade

\begin{tabular}{ccc}
\hline & M & DP \\
\hline Homens & 81 & 21 \\
Mulheres & 83 & 7.1 \\
Total & 82.5 & 13.2 \\
\hline
\end{tabular}

Tabela - 2: Caracterização da amostra em função do gênero, estado civil, nível de escolaridade e agregado familiar

\section{Variáveis}

Rua São Francisco Xavier, 524, $2^{\circ}$ andar - BL D, sl 2025 - Maracanã 


\section{Gênero}

Masculino

Feminino

\section{Estado Civil}

Solteira(o)

Casada(o)

Separada(o)

Viúva(o)

União de fato

\section{Nível de Escolaridade}

Sem escolaridade

Até 4 anos de escolaridade

Outro

\section{Reside Sozinho}

Sim

Não
16

34

$32 \%$

$68 \%$

12

1

31

1

$10 \%$

$24 \%$

$2 \%$

$62 \%$

$2 \%$

27

$54 \%$

16

$32 \%$

7

$14 \%$

22

$43 \%$

28

$55 \%$

Pode-se constatar que se trata de uma amostra com indivíduos maioritariamente do gênero feminino (68\%), viúvos (62\%) e que não têm escolaridade (54\%). Do total, 55\% das pessoas idosas não residem sozinhas. A que se referir que estes participantes até podem não residir sozinhos, mas não invalida que não estejam sozinhos na maior parte do tempo.

O questionário de dados sociodemográficos foi, ainda, constituído com questões específicas, formuladas de acordo com o contexto e o objetivo de toda a investigação, Servindo também para se tirar conclusões mais precisas sobre o suporte social de amigos e vizinhos (c.f. Tabela 3).

Tabela - 3: Resultados relativos às questões específicas dos questionários da amostra

\begin{tabular}{lccc}
\hline & & N & $\%$ \\
\hline Considera que os seus amigos e/ ou & Sim & 48 & $96 \%$ \\
vizinhos lhe prestam o auxílio & Não & 2 & $4 \%$ \\
\hline
\end{tabular}

\section{POLÊM!CA | Rerisa Eetronica da ver}




\section{necessário?}

Sente-se protegido pelos seus amigos/

vizinhos?

$\begin{array}{ccc}\text { Sim } & 49 & 98 \% \\ \text { Não } & 1 & 2 \% \\ \text { Amigos/ } & & \\ \text { Vizinhos } & 25 & 50 \% \\ \text { Familiares } & 25 & 50 \%\end{array}$

Constata-se que as pessoas idosas isoladas consideram que os amigos e/ou vizinhos lhe prestam o apoio necessário (96\%) e que estes se sentem protegidos pelos amigos/ vizinhos (98\%). Um dado curioso observado na tabela anterior refere-se ao apoio tanto dos amigos como dos familiares, quando um idoso necessita de alguma coisa urgente, sendo que com os dados apresentados não se conseguem tirar conclusões uma vez que metade da amostra (50\%) referem recorrer aos amigos/vizinhos e a outra metade aos familiares.

\section{Escala Satisfação com o Suporte Social (ESSS)}

A partir dos dados obtidos (c.f. Tabela 4), pode-se verificar que, em média, os participantes apresentam uma pontuação total da ESSS de 55,54 pontos $(D P=8.3)$, sendo que a população masculina obteve uma pontuação total de $57.5(D P=8.4)$ e a população feminina $54.6(D P=8.3)$. Este resultado indica que a percepção da ESSS na pontuação total no caso das redes de amigos e vizinhos são um bom nível de apoio para as pessoas idosas isoladas, uma vez que a amplitude da pontuação total da ESSS, varia entre 15 e 75 . Uma pontuação mais alta corresponde a uma percepção de maior suporte social.

Tabela - 4: Médias e desvios-padrão da pontuação total da ESSS em função do gênero

\begin{tabular}{cccc}
\hline & M & DP & A \\
\hline Homens & 57.5 & 8.4 & $42-71$ \\
Mulheres & 54.6 & 8.3 & $34-68$ \\
Total & 55.54 & 8.3 & $34-71$ \\
\hline
\end{tabular}

\section{POLÊM!CA | Revista Eletronica da veri}


Pode-se, ainda, verificar que a pontuação mínima obtida no conjunto da amostra foi de 34 pontos, e a máxima de 71. A amplitude mínima para a população masculina foi de 42 pontos e a máxima de 71 pontos. Relativo ao gênero feminino, observa-se uma pontuação mínima de 34 pontos e uma máxima de 68 pontos.

Para além da pontuação total da ESSS, esta é constituída por quatro fatores (subescalas), sendo a pontuação total de cada uma apresentada seguida após regra de três simples ${ }^{1}$.

Tabela - 5: Pontuação totais obtidas nas 4 dimensões da ESSS

\begin{tabular}{lc}
\hline \multicolumn{1}{c}{ Fatores/ Subescalas } & Pontuações obtidas (0 a 100) \\
\hline Satisfação com os amigos (SA) & 79,44 \\
Intimidade (IN) & 74,20 \\
Satisfação com a família (SF) & 81,61 \\
Atividades Sociais (AS) & 57,50 \\
\hline
\end{tabular}

Com os resultados obtidos, verifica-se que todos os fatores apresentam valores acima da média (50 pontos), sendo que a subescala "Atividades Sociais" (AS) é o domínio que apresenta uma pontuação mais baixa comparativamente com os restantes fatores $(57,50)$. Em contrapartida, os fatores que apresentam uma pontuação mais elevada são a "Satisfação com a Família" (81,61) e "Satisfação com os Amigos" (SA) $(79,44)$, não existindo diferença significativa entre estas duas variáveis. Outro dado apresentado é o fator "Intimidade" (IN) apresentando uma pontuação de $(74,20)$, verificando-se que no que concerne ao suporte social, a população idosa do estudo indica que, para além das relações familiares e de amizade, o suporte íntimo é o que lhes proporciona maior satisfação.

\section{Discussão}

O presente estudo pretendeu perceber efetivamente se os amigos/ vizinhos são um bom suporte social para as pessoas idosas que vivem isoladas. Neste sentido, e de acordo com

\footnotetext{
${ }^{1}$ Dado que, o número de itens de cada dimensão é diferente, as pontuações mínimas e máximas por dimensão também o são, como já referido nos métodos. Recorreu-se à regra de três simples para se converter as pontuações obtidas entre 0 e 100 , sendo que $0=$ pontuação mais baixa possível e 100 = pontuação mais elevada possível.
} 
os resultados obtidos através da ESSS, verificou-se que os vizinhos e/ou amigos são um bom nível de apoio para as pessoas idosas isoladas, confirmando-se a hipótese formulada. Este resultado vai ao encontro dos estudos desenvolvidos por Sequeira e Silva (2002), que tinham como principais objetivos perceber quais eram os fatores inerentes à satisfação num grupo de residentes em meio rural. O mesmo estudo foi constituído por 50 participantes idosos, concluindo que em contexto de meio rural existem níveis de bem-estar apontando-se para uma adequada relação idoso/meio.

Relativamente ao sentimento de segurança e de proteção que as pessoas idosas possam sentir, esta hipótese é confirmada através das pontuações obtidas nas questões específicas do questionário elaborado para a presente investigação. Através da análise de resultados verificou-se que $96 \%$ da amostra considera que os amigos prestam o auxílio necessário. Para além da presente investigação, estudos diversos ajudam a confirmar este resultado. No estudo desenvolvido por Cabral e colaboradores (2013), o mesmo possibilitou avaliar as relações de vizinhança, tendo em consideração três aspetos: identificação dos vizinhos, a frequência de conversação e o tempo de permanência. Através dos resultados obtidos pode-se verificar que os mesmos permitem deduzir um elevado conhecimento, ou pelo menos reconhecimento dos vizinhos e um padrão bastante regular de interações sociais. Relativamente à proteção das pessoas idosas isoladas por parte dos amigos e/ou vizinhos, verificou-se que $98 \%$ da amostra refere sentir-se protegido. Muitas das vezes o que se verifica é que a proteção em meio rural está muito associada às relações de confiança que vão sendo estabelecidas pelos diversos elementos. Este mesmo resultado e conclusão estão de acordo com o estudo desenvolvido por Franquinello e Marcon (2011), que tinha como principal objetivo compreender a atuação da rede representada pelos amigos e vizinhos de adultos idosos hipertensos. As conclusões do estudo reportam para o fato de o tempo de moradia interferir no vínculo com os vizinhos.

Além dos resultados apresentados indicarem que as redes de amigos e vizinhança são um bom suporte social e de proteção para pessoas idosas isoladas/meio rural, existe por parte destes uma distinção entre o que para eles são amigos e vizinhos. Estas mesmas conclusões só foram possíveis através do contato pessoal com pessoas idosas isoladas, onde estes afirmam muitas das vezes que até podem ter vizinhos, mas que estes não são amigos. Esta mesma conclusão poderá estar associada à confiança entre as pessoas idosas em meio isolado.

\section{POLÊM!CA | Revista Eletronica da Uej}


No que diz respeito à hipótese das pessoas idosas residentes em meio rural apresentarem uma menor satisfação relativa aos seus amigos comparativamente à família, os resultados obtidos no estudo apontam para a inexistência de uma diferença significativa. Assim, não se conseguiu comprovar que as pessoas idosas apresentam uma maior satisfação em relação à família do que em relação aos amigos. Por outro lado, os dados apresentados podem querer dizer que a família e os amigos são o principal suporte social para as pessoas idosas isoladas e que os mesmos se complementam, ou interligam. Através do questionário sociodemográfico, pode-se verificar esta mesma semelhança, uma vez que 50\% da amostra refere recorrer aos amigos e/ou vizinhos em caso de necessidade urgentes e os outros 50\% recorrem aos familiares. Isto vai ao encontro do estudo desenvolvido por Leite, Battista, Berlezi e Schever (2008) tendo como principal objetivo analisar o suporte familiar e social de 338 pessoas idosas, com uma faixa etária dos 60 aos 65 anos.

As conclusões do estudo indicam que $87 \%$ das pessoas idosas possuem uma rede familiar e social que lhes fornecem suporte de natureza emocional, material, afetiva e informativa. No caso de a família dos idosos isolados e residentes em meio rural não prestarem o apoio necessário, são os amigos e/ou vizinhos que assumem este papel, sendo o principal meio de ajuda. Isto mesmo pode-se verificar, por exemplo, no caso de as pessoas idosas terem familiares no estrangeiro, ou então na ausência por determinados períodos de tempo (ex. trabalho na cidade). No estudo desenvolvido por Fonseca, Paúl, Martin e Amado (2005) onde numa amostra de 117 indivíduos, com uma média de idades de 74,5, concluiu-se que as pessoas idosas rurais envelhecem longe dos filhos e netos.

\section{Conclusão}

No início desta investigação houve uma questão que esteve na base de todo o trabalho, a de saber até que ponto a rede de amigos e vizinhança contribuía para a satisfação e ao mesmo tempo para a proteção social de pessoas idosas residentes isoladas e/ou meio rural.

De todos os dados demográficos pode-se concluir que o perfil dos participantes no estudo caracteriza-se por serem, na sua maioria, do gênero feminino, com idade média de 82 anos, viúvos e que se sentem protegidos pelos amigos e/ou vizinhos, que prestam-lhe o auxílio necessário.

\section{POLÊM!CA | Revista Eletronica da Ueij}


Relativamente à ESSS, verifica-se que os vizinhos e/ou amigos são um bom nível de apoio para as pessoas idosas isoladas $(M=55,54)$. A variável Satisfação com os Amigos (SA) obtém uma pontuação inferior, comparativamente com a variável Satisfação com a Família (SF), 79,44 e 81,61 pontos, respectivamente.

Partindo da questão colocada, conclui-se que não há uma diferença significativa que possa comprovar que as pessoas idosas apresentam uma maior satisfação em relação à família do que em relação aos amigos, ou seja, ambos se complementam no cuidado à pessoa idosa.

De acordo com os fatos, o presente trabalho apresenta algumas limitações, que devem ser consideradas em investigações futuras, nomeadamente: o tamanho da amostra deveria ser maior de forma a ser mais representativa da população idosa do concelho abrangido, tirandose conclusões mais profundas relativas às hipóteses formuladas.

Atualmente, 377 pessoas idosas vivem isoladas e sozinhas no Concelho da Guarda, neste sentido e como o fim de um trabalho é sempre uma oportunidade da realização de um novo, fica a questão: serão as redes de amigos e vizinhos nas pessoas idosas mais eficazes nas aldeias do que nas Cidades?

\section{Referências}

CABRAL, M. (Org.), FERREIRA, P., SILVA, P., JERÔNIMO, P., \& MARQUES, T. Processos de envelhecimento em Portugal, uso do tempo, redes socais e condições de vida. Lisboa: Fundação Francisco Manuel dos Santos, 2013.

DOMINGUES, M. Mapa mínimo de relações do idoso: uma ferramenta para avaliar rede de suporte social. In: PEREIRA, F. (Org.). Teoria e prática da gerontologia: um guia para cuidadores de idosos. (pp.175-188). Viseu: Psicosoma, 2012.

FAQUINELLO, P. \& MARCON, S. Amigos e vizinhos: uma rede social ativa para adultos e idosos hipertensos. Rev. Esc. Enferm. USP, 45(6), 345-52, 2011.

FONSECA, A., PAÚL, C., MARTIN, I. \& AMADO, J. Condição psicossocial de idosos rurais numa aldeia do interior de Portugal. In: PAÚL, C. \& FONSECA, A. (Cord.). Envelhecer em Portugal: psicologia, saúde e prestação de cuidados (pp. 97-108). Lisboa: Climepsi Editores, 2005.

LEITE, M., BATTIST, I., BERLEZI, E. \& SCHEUER, A. Idosos residentes em meio urbano e sua rede de suporte familiar e social. Texto \& Contexto-Enfermagem, 17(2), 250-257. 2008.

MONTEIRO, C. (2012). Idosos residentes em meio rural: satisfação com o suporte social. Tese de mestrado não publicada apresentada ao Departamento de Economia, Gestão e Ciências Sociais, Universidade Católica Portuguesa.

SEQUEIRA, A. \& SILVA, M. O bem-estar da pessoa idosa em meio rural. Análise Psicológica, 20(3), 505-516. 2002. 
RIBEIRO, José L. P. Escala de satisfação com o suporte social (ESSS). Análise Psicológica,17(3), 547-558. 1999.

Recebido em: 02/07/2015.

Aceito em: 29/07/2015. 